Paper

\title{
Noise reduction and signal enhancement by receptor synchronization
}

\author{
Seido Nagano ${ }^{1 a)}$ \\ ${ }^{1}$ Department of Bioinformatics, Ritsumeikan University \\ 1-1-1 Nojihigashi, Shiga 525-8577, Japan \\ a)nagano@sk.ritsumei.ac.jp
}

Received February 6, 2020; Revised April 23, 2020; Published October 1, 2020

\begin{abstract}
The ligand-receptor interaction is critical for signal transduction in biological systems. The binding interaction between a receptor and its cognate ligand has been extensively investigated at the molecular level. However, there are often many non-ligand molecules surrounding receptors, and it is very likely that they function as extraneous noise that disrupts authentic signal transduction. Herein we demonstrated that excitable receptor synchronization during signal transduction is key for noise reduction and signal enhancement.
\end{abstract}

Key Words: noise-reduction, signal-enhancement, receptor, synchronization, excitability, chemotaxis

\section{Introduction}

The ligand-receptor interaction is essential for signal transduction in biological systems. Multiple studies have investigated ligand-receptor interactions from a variety of perspectives [1] including biochemical association-dissociation relationships, as molecular conformations, in chemotactic processes [2], and as receptor-receptor interactions [3]. Nonetheless, our understanding of receptor dynamics is still not satisfactory due to the complexity of surrounding biological molecules in the environment. For example, there are both ligand molecules and other non-ligand molecules, including water molecules, in the environment surrounding the receptors. Besides, there are often several thousand receptors of the same type within a cell. Thus, there is ample opportunity for non-ligand molecules to disrupt proper ligand-mediated signal transduction as shown in Fig. 1(b).

To date, the underlying mechanism responsible for the coordination of receptor signaling within a

(a)

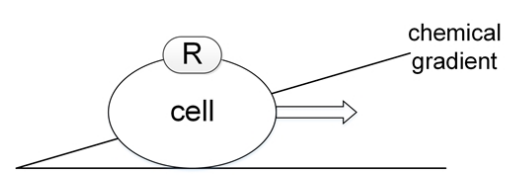

(R) : receptor (b)

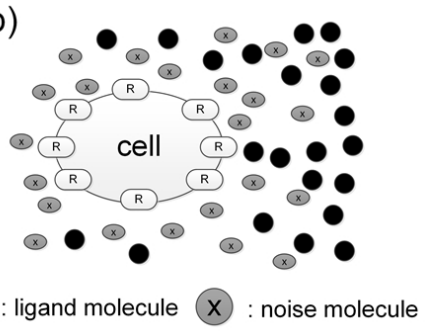

Fig. 1. Chemotaxis. (a) single receptor model. (b) real situation. 
whole cell has not been seriously investigated, except in Escherichia coli. Receptor interactions in E. coli chemotaxis have been extensively investigated in the context of the enhancement of ligand sensitivity $[4,5]$. The existence of extraneous noise may be a greater problem in environments with a small ligand density gradients as shown in Fig. 2(b). In E. coli, even a small ligand density gradient is readily detectable [6]. Therefore, there must be some type of noise reduction mechanism in place to preferentially enhance only bona fide ligand-receptor signals.

\section{(a)}

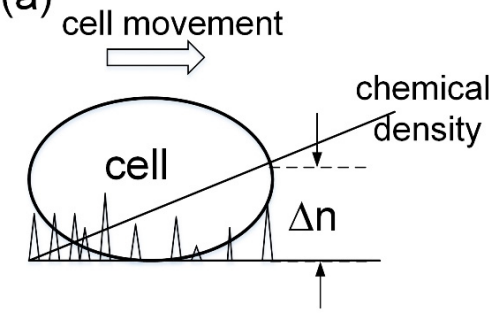

$\Delta \mathrm{n}>\mathrm{n}_{\text {noise }}$ (b)

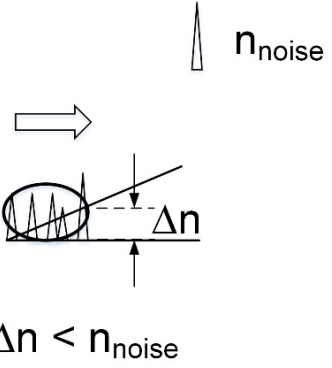

Fig. 2. Cell size dependence of the relationship between the detectable chemical density difference $\Delta n$ and the random noise $n_{n o i s e}$, where (a) $\Delta n>n_{\text {noise }}$, and (b) $\Delta n<n_{\text {noise }}$.

We hypothesized that synchronization between receptors works as a noise-reduction system. In order to investigate ligand-receptor signaling in a small ligand density gradient, we used the cellular slime mold Dictyostelium discoideum [7]. When starved, Dictyostelium secrets adenosine 3', 5'monophosphate (cAMP) pulses with a periodicity of 5-10 minutes to the outside of the cell, and this stimulates chemotaxis along the cAMP gradient. Dictyostelium express thousands of cAMP receptors, which makes it the model system for the investigation of noise-reduction by receptors. Based on our understanding of the molecular network for the spontaneous production of cAMP $[8$, 9], as well as the excitable property of the cAMP receptor, we studied how receptors can respond to specific signals amongst environmental noise.

\section{Analysis}

\subsection{Hypothesis of noise reduction by receptors}

In this study we sought to determine the mechanism that allowed receptors to distinguish between signal and noise. This is particularly important when the ligand molecules may serve as real messenger molecules. We assume here that noises are detected at random by receptors and they are not excited, while the real signal ligand molecules simultaneously induce excitement of nearby receptors

(a)

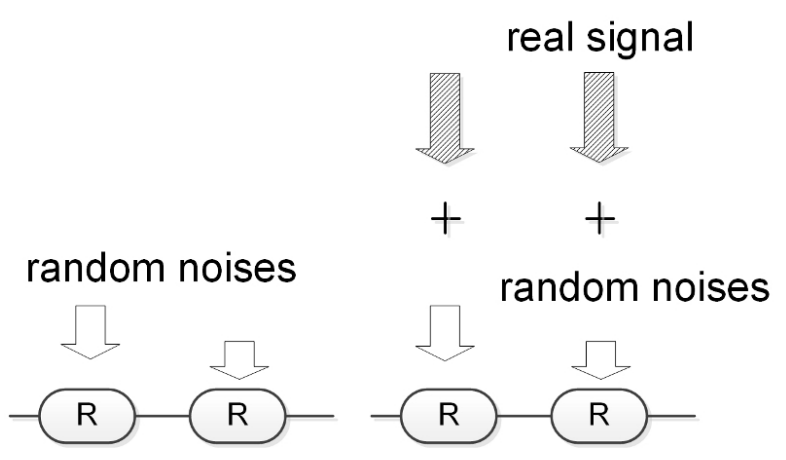

Fig. 3. Hypothesis. (a) Noises arrive in different receptors at different times. (b) Superposition of the real signal and random noises. Real signals arrive in nearby receptors simultaneously, but noises do not. 
and internal signal enhancement is achieved. In other words, a single receptor cannot distinguish between real signal and noise. We also assume here that time adjustment is achieved by synchronization between excited receptors.

\subsection{Three-variable receptor model}

Spontaneous cAMP production of Dictyostelium discoideum amoeba is a well known phenomenon. Recently, we have successfully explained them by the molecular network presented in Fig. 4.

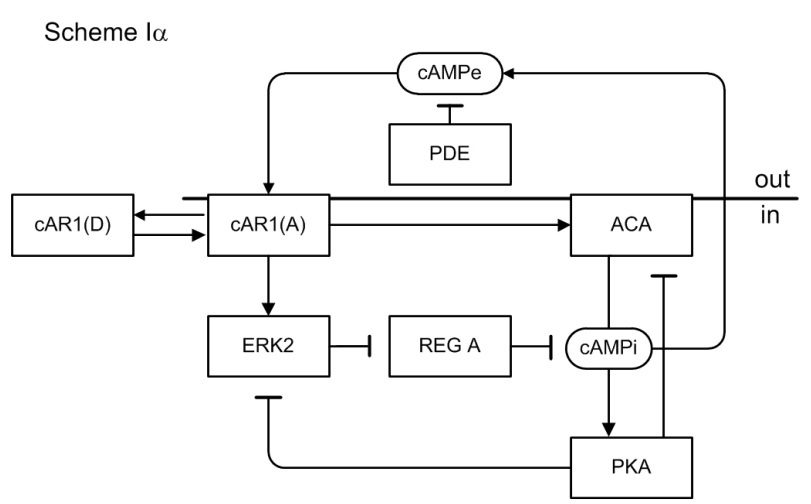

Fig. 4. A molecular network for spontaneous cAMP production of Dictyostelium amoeba [8].

When the extracellular cAMP (cAMPe) binds to the cAMP receptor (cAR1) of a Dictyostelium amoeba, adenyl cyclase (ACA) and the mitogen-activated protein (MAP) kinase (ERK2) are activated by the ligand-bound receptor cAR1. Intracellular cAMP (cAMPi) activates cAMP-dependent protein kinase A (PKA) and it inhibits ERK2, leading to a loss-of-ligand binding by cAR1 due to the inhibition of the inhibitor ERK2. ERK2 phosphorylates the intracellular phosphodiesterase (Reg A). The cAMPi is secreted to the outside of the cell as cAMPe at the rate $k_{L}$, and then PDE phosphorylates cAMPe. This signaling cascade initiates spontaneous cAMP oscillation [8].

To elucidate essence of the complex molecular network, we simplify it as a three-variable receptor model as shown in Fig. 5.

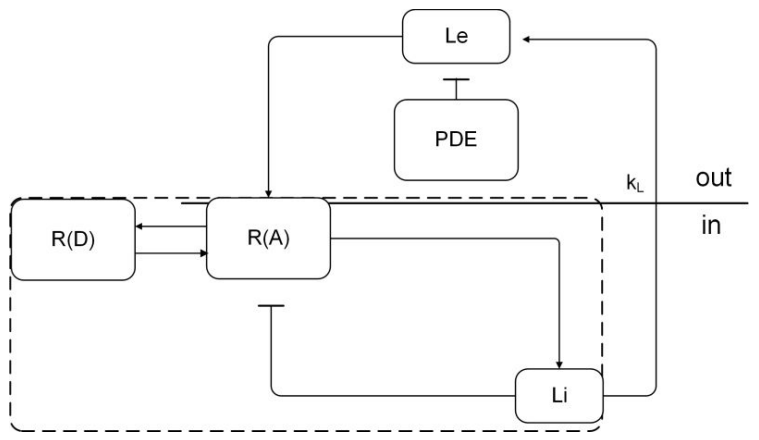

Fig. 5. Three-variable receptor model. When the extracellular ligand (Le) binds to the receptor $(\mathrm{R}), \mathrm{R}$ activates the production of the intracellular ligand ( $\mathrm{Li}$ ), and $\mathrm{Li}$ suppresses the activity of $\mathrm{R}$. The $\mathrm{Li}$ is also secreted to the outside of the cell (Le) at the rate $k_{L}$. PDE phosphorylates Le.

The scheme is summarized as the following mathematical expression:

$$
\begin{aligned}
& \frac{d[L i]}{d t}=k_{a}[R]-k_{b}[L i] \\
& \frac{d[L e]}{d t}=k_{L}[L i]-k_{c}[L e]
\end{aligned}
$$




$$
\frac{d[R]}{d t}=k_{d} R_{\alpha}([L e])-k_{e}[L i][R],
$$

where $R_{\alpha}([L e])$ is the receptor function with adaptation defined as

$$
R_{\alpha}([L e])=\frac{[L e]}{1+\left([L e] /[L e]_{c}\right)^{\alpha}} .
$$

With an increase of $[L e], R_{\alpha}([L e])$ increases from zero, but it starts to drop down to zero when $[L e]$ become larger than $[L e]_{c}$. This experimentally observed characteristic originates in the G-protein dynamics [9]. There are eight parameters in Eqs. (1) to (3). Specifically, $k_{L}$ is the leak rate of ligand molecules from the cell. Figure 6 shows two examples with different of $k_{L}$ values. Spontaneous oscillation is undetectable when $k_{L}=0.1$, but becomes apparent when $k_{L}=0.5$.

Table I. Parameters of the three-variable receptor model. Spontaneous oscillation appears when $\underline{k}_{j} \leq k_{j} \leq \bar{k}_{j}$.

\begin{tabular}{|c|c|c|c|}
\hline parameter & adopted value & $\underline{k}_{j}$ & $\bar{k}_{j}$ \\
\hline $\mathrm{k}_{a}$ & 5.2 & 0.68 & $>200$ \\
\hline $\mathrm{k}_{b}$ & 0.4 & 0.10 & 1.68 \\
\hline $\mathrm{k}_{L}$ & 0.5 & 0.19 & 2.47 \\
\hline $\mathrm{k}_{c}$ & 0.9 & 0.11 & 2.01 \\
\hline $\mathrm{k}_{d}$ & 0.9 & 0.12 & $>200$ \\
\hline $\mathrm{k}_{e}$ & 0.1 & 0.02 & 0.44 \\
\hline$[L e]_{c}$ & 2.0 & 0.45 & 8.84 \\
\hline$\alpha$ & 20.0 & 11.98 & $>200$ \\
\hline
\end{tabular}

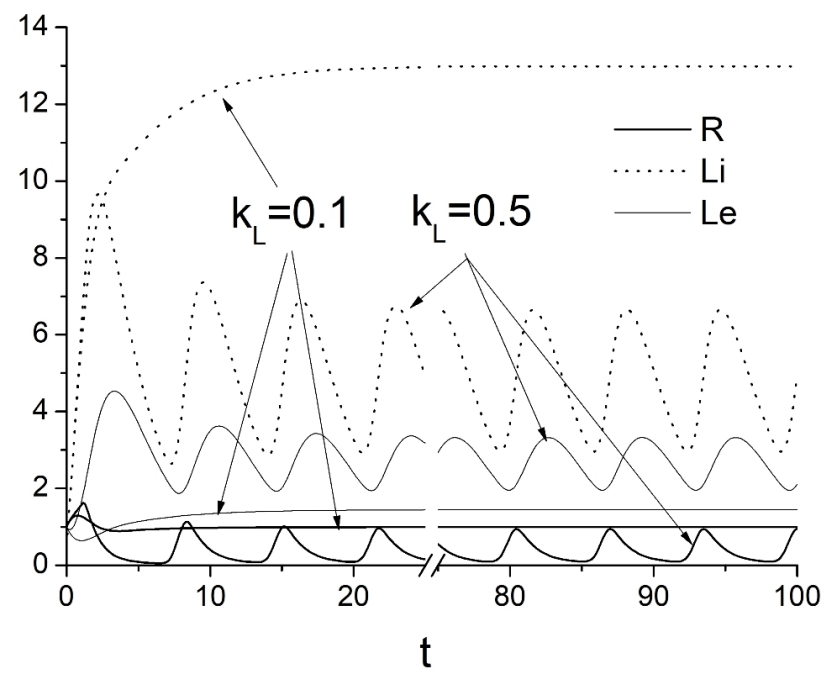

Fig. 6. Spontaneous oscillations appear when $0.19 \leq k_{L} \leq 2.47$ as shown in Table I, where $k_{L}$ is the leak rate of the ligand molecules. $\mathrm{R}, \mathrm{Li}$, and Le are receptor activity, intracellular ligand density, and extracellular ligand density, respectively.

\subsection{Synchronization between receptors within a cell}

We have proposed the theory of diffusion-assisted synchronization between cells[10]. Furthermore, we have mathematically generalized it as the receptor-product coupling scheme for synchronization [11, 12], and it enabled the mutual synchronization between general limit cycle oscillators. Following our previous works, we generalize current single receptor model to multi-receptor systems. The scheme is summarized as the following mathematical expression: 


$$
\begin{aligned}
\frac{d[L i]_{j}}{d t} & =k_{a}[R]_{j}-k_{b}[L i]_{j}, \\
{[L i S]_{j} } & =[L i]_{j}+d \sum_{i \neq j}^{N}[L i]_{i}, \\
\frac{d[L e]_{j}}{d t} & =k_{L}[L i S]_{j}-k_{c}[L e]_{j}, \\
\frac{d[R]_{j}}{d t} & =k_{d} R_{\alpha}\left([L e]_{j}+\left[L_{e x t}\right]_{j}+\left[L_{\text {noise }}\right]_{j}\right)-k_{e}[L i]_{j}[R]_{j}, \\
{\left[L_{\text {noise }}(t)\right]_{j} } & =\sum_{i}^{n_{\text {rand }}} A_{i j}^{\text {rand }} \exp \left[-\beta\left(t-t_{i j}^{\text {rand }}\right)^{2}\right],
\end{aligned}
$$

where $d$ is the intracellular ligand diffusion constant, $\mathrm{L}_{e x t}$ is the real extracellular ligand signal, and $\mathrm{L}_{n o i s e}$ is the extracellular noise signal. We assume that the noise signal with random strength $A_{i j}^{\text {rand }}$ approaches the receptor $j$ at random time $t_{i j}^{r a n d}$. We have also adopted the Gaussian function with $\beta=200$ to reproduce noise character.

Figure 7 shows our scheme for two receptors. Figure 8 shows a typical case of synchronization between two receptors in the absence of external signal and noises.

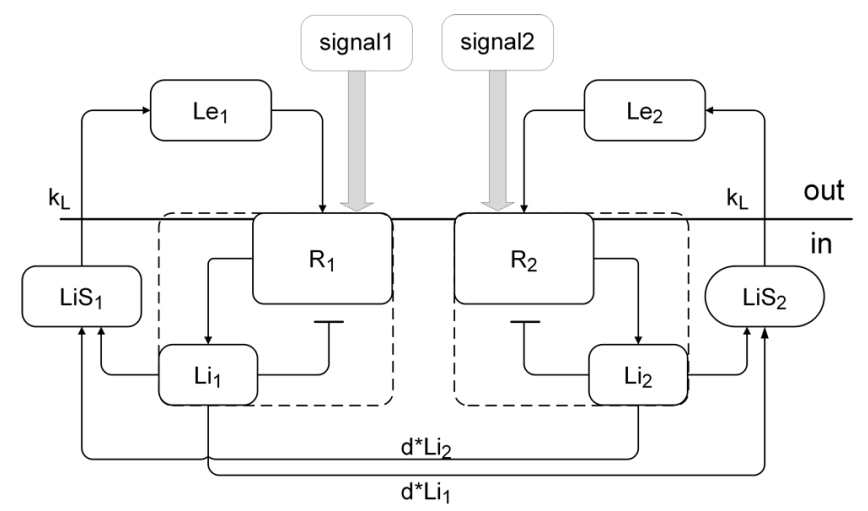

Fig. 7. Coupling scheme between two receptors by intracellular ligand diffusion as presented in Eqs. (5) to (9). Here $d$ is the diffusion constant of intracellular ligand. Thus, $d<1$. LiS is the combined intracellular ligand density; namely $\mathrm{LiS}_{1}=\mathrm{Li}_{1}+\mathrm{d} \cdot \mathrm{Li}_{2}$, and $\mathrm{LiS}_{2}=\mathrm{Li}_{2}+\mathrm{d} \cdot \mathrm{Li}_{1}$.

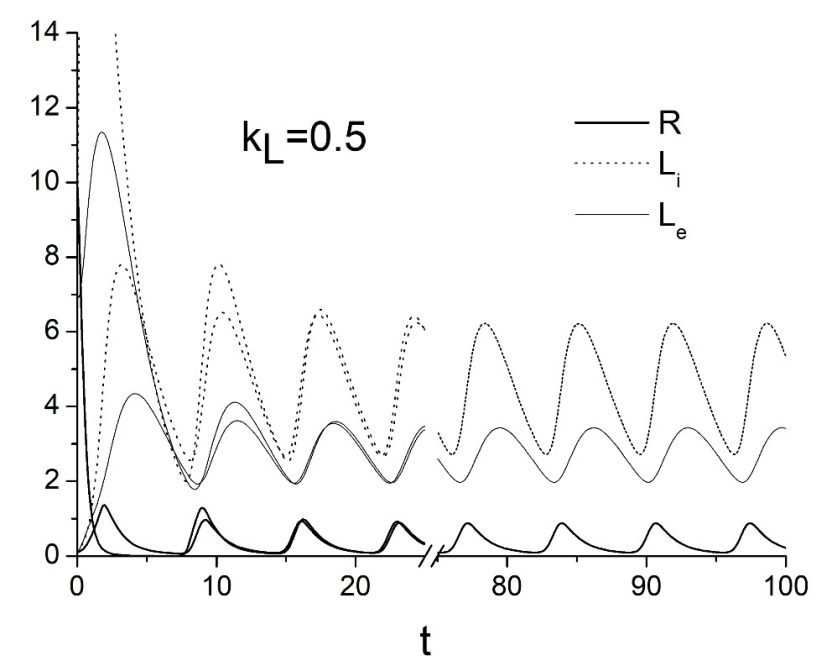

Fig. 8. Synchronization between two receptors in a cell when $k_{L}=0.5$. 


\subsection{Excitability of multi-receptor systems}

Although a single receptor failed to show spontaneous oscillation when $k_{L}<0.19$, as the number of receptors $n_{r}$ increased we observed an increase in spontaneous oscillation. Thus, we determined that $d, n_{r}$, and $k_{L}$ were mutually related, and the synchronous spontaneous oscillation appeared under the following condition:

$$
d \cdot n_{r} \cdot k_{L} \gtrsim a-b \cdot k_{L}
$$

is satisfied as shown in Fig. 9, where $a=0.192, b=0.887$.

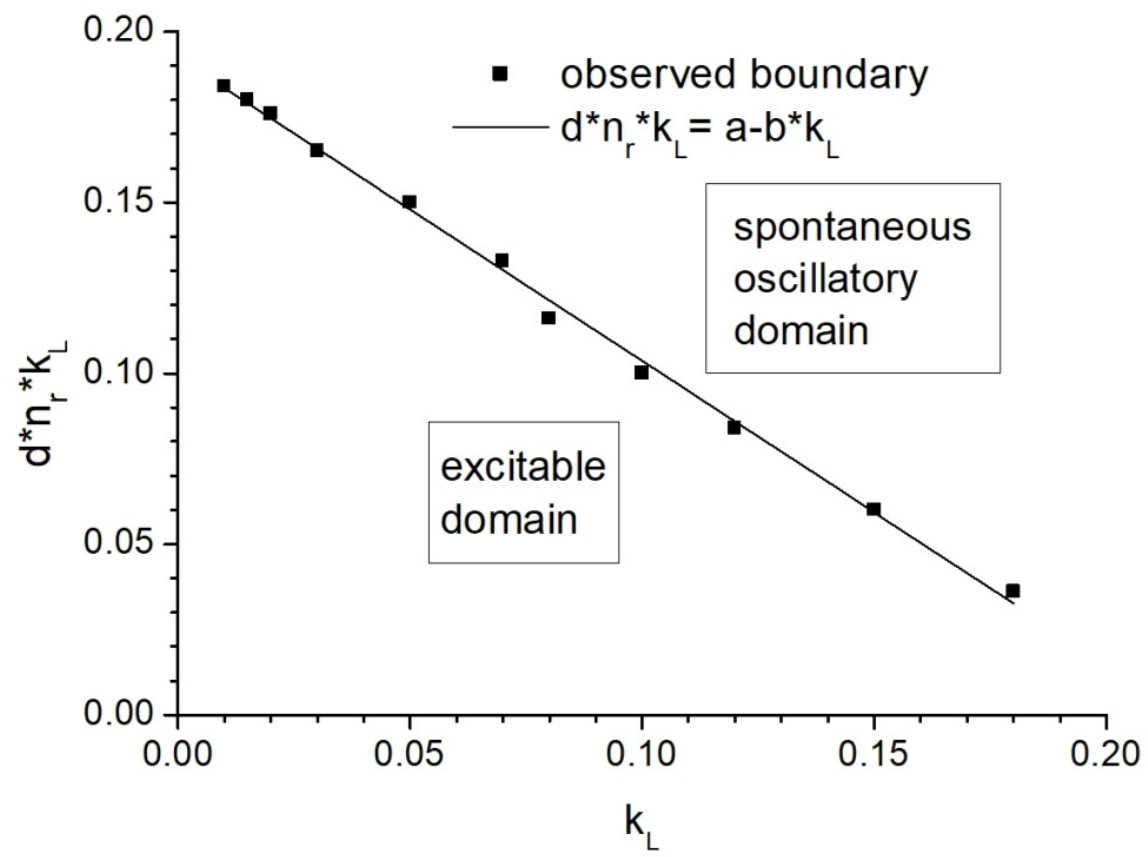

Fig. 9. Excitability of multi-receptor systems. Spontaneous oscillations were not detected for a single receptor when $k_{L}<0.19$. However, synchronous spontaneous oscillation appear when $d \cdot n_{r} \cdot k_{L} \gtrsim a-b \cdot k_{L}$, where $a=0.192$ and $b=0.887$, as the number of receptors $n_{r}$ increases.

Figure 9 shows that intracellular coupling between receptors enhanced the excitability of a cell. When the leak rate $k_{L}$ is small, receptor synchronization is achieved only if the number of receptors $n_{r}$ is sufficiently large.

\subsection{Noise reduction and signal enhancement}

In order to investigate the effect of synchronization between receptors, we applied random noises $\mathrm{L}_{\text {noise }}$ when $30 \leq t \leq 60$, and a real signal $\mathrm{L}_{e x t}=a(1+\sin \omega t)$, which mimics the experimentally observed periodic chemical signal, when $50 \leq t \leq 70$ with $a=0.3$ and $\omega=1.0$. As shown in Fig. 10, based on the $\mathrm{Li}$ a single receptor failed to distinguish between noise and a real signal. However, noise was reduced to one third, and the real signal was enhanced 17 times in Li when the number of receptors is 10 in the excitable domain (Fig. 11). Saturation of the signal enhancement in Li was observed when the number of receptors was further increased as well as the spontaneous oscillatory domain (Fig. 12). The maximum amplitude of noises was chosen 10 times larger than that of the real signal in Figs. 10, 11, and 12.

\section{Conclusions}

Let me briefly summarize our findings here. (a) A receptor network system in Fig. 5 constitutes a limit cycle oscillator when $0.19 \leq k_{L} \leq 2.47$ from Table I. (b) Fig. 8 shows that synchronization between receptors can be achieved by the diffusion of intracellular molecules when $0.19 \leq k_{L} \leq 2.47$. (c) Fig. 10 shows that single receptor system cannot be excited by the external stimulus when $k_{L}<0.19$. 


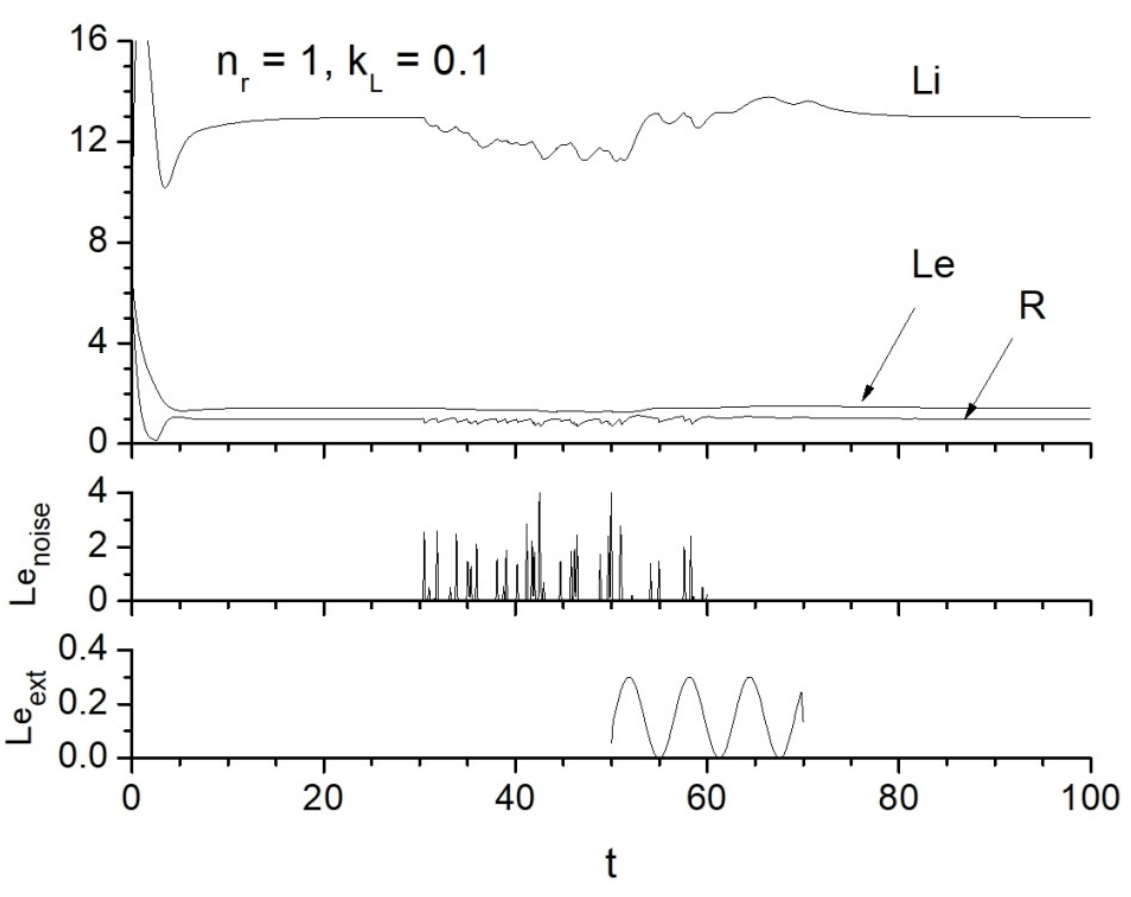

Fig. 10. Single receptor system with $k_{L}=0.1$. Random noises with $A_{i j}^{\text {noise }}<$ 3 were applied when $30 \leq t \leq 60$, and real signal $a(1+\sin \omega t)$ with $a=0.3$ and $\omega=1.0$ was applied when $50 \leq t \leq 70$. Real signal and noises were mixed up in Li.

\section{excitable domain}

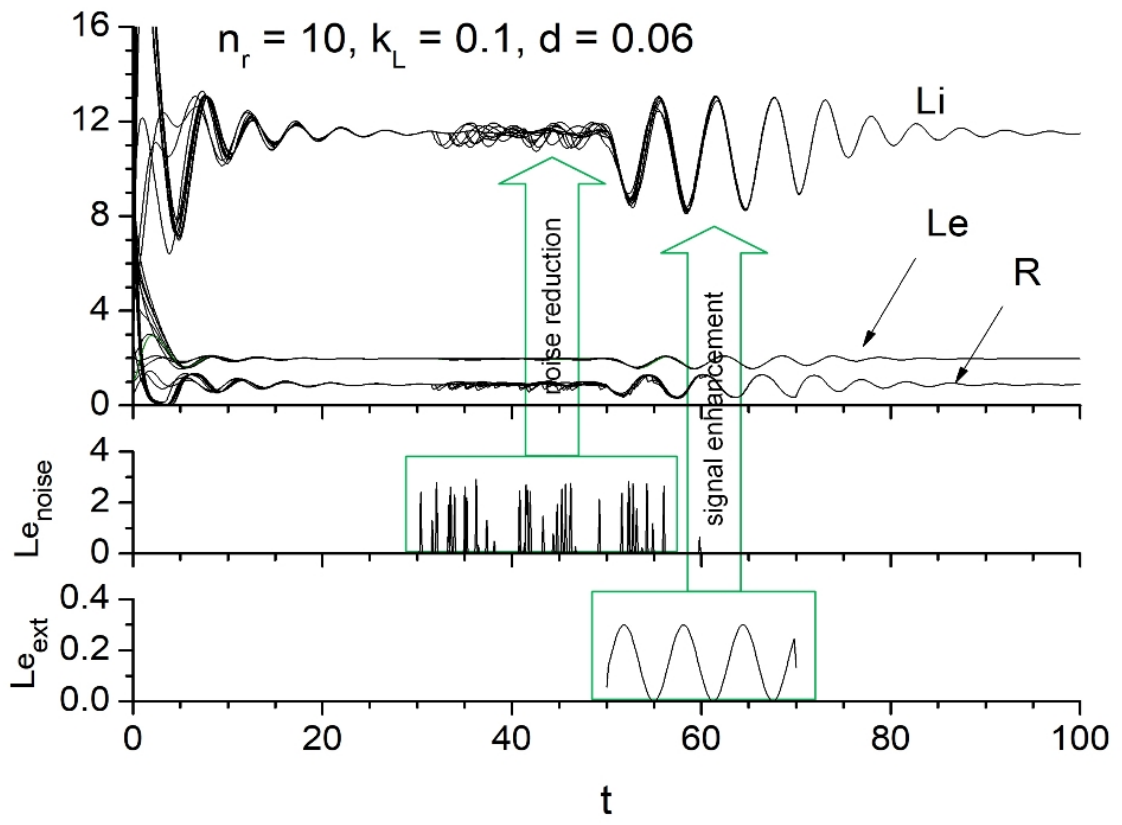

Fig. 11. Ten receptor system with $k_{L}=0.1$, and $d=0.06$, where $d \cdot n_{r} \cdot k_{L}=$ $0.06<a-b \cdot k_{L}$ (excitable domain). Within a cell, the real signal was enhanced 17 times in $\mathrm{Li}$ and noises were reduced to one third.

This characteristics can work as noise reduction. (d) Although a receptor network system in Fig. 5 becomes a damped oscillator when $k_{L}<0.19$, the coupled receptor system can become a limit cycle oscillator by increasing number of joining receptors under the same condition as shown in Fig. 9. (e) When number of coupled receptors is not so large, they stay in the excitable domain as shown in 


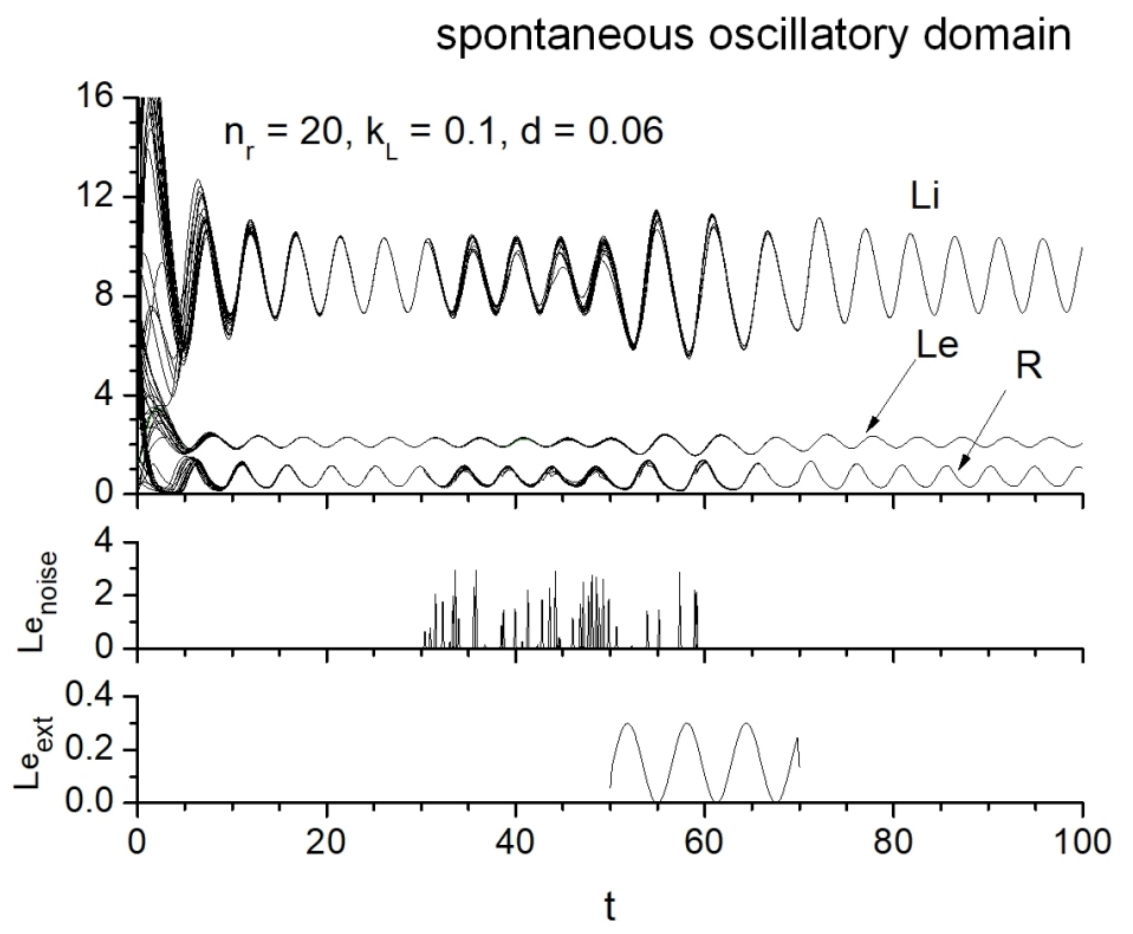

Fig. 12. Twenty receptor system with $k_{L}=0.1$, and $d=0.06$, where $d \cdot n_{r}$. $k_{L}=0.12>a-b \cdot k_{L}$ (spontaneous oscillatory domain). Saturation of signal enhancement in Li is observed here. Besides, separation between real signal and noise is difficult.

Fig. 9. But coupled receptor system can be excited and mutual synchronization is achieved at the same time when they are simultaneously stimulated. As a result, signal enhancement is achieved as shown in Fig. 11. (f) In the spontaneous oscillatory domain, noise reduction is observed, but a significant signal enhancement was not observed as shown in Fig. 12.

The above findings are for a single cell system. However, we have observed similar excitable properties in the multi-cellular systems of Dictyostelium amoeba [9,10]. Intracellular biochemical reactions between cells were mutually synchronized via receptors and secreted extracellular cAMP. There was also a threshold number of cells for the sustained spontaneous cAMP oscillations. When number of cells in a cluster is small, no spontaneous oscillation was observed. But as the size of cluster increased, pulsatile cAMP oscillations were initiated. Some of their snapshots are presented in Appendix A.

In biological systems a persistent challenge is how to decipher meaningful information amongst environmental noise. We sought to investigate this issue using a biological system with a small ligand gradient to model the ability of cells to respond to specific stimuli under limiting conditions. Consistent with our hypothesis, the separation of the ligand-receptor signals and environmental noise required the expression of multiple receptors. Furthermore, optimal receptor signaling was achieved by receptor synchronization. It is important to keep in mind that random noise could not exceed the threshold for receptor excitability. Significant enhancement of the external signal was achieved via simultaneous receptor signaling that initiated the onset of spontaneous oscillation. This current study largely recapitulated the findings of a significant enhancement of sensing in $E$. coli. The arrival of ligands and the synchronous excitability of the receptors worked together to amplify the small ligandmediated signal amongst the environmental noise. Taken together our findings demonstrated cellular mechanisms that functioned together as a noise reduction filter during biological signal transduction.

\section{Acknowledgments}

We appreciate Dr. Shunsuke Sakurai for the determination of rate constant values presented in Table I. 
We also appreciate Mr. Hiroyuki Tabira and Mr. Yuuki Shimizu for various numerical confirmations.

\section{Appendix}

\section{A. Excitability of multi-cellular systems [9]}

Cellular dynamics simulation study confirmed the existence of a threshold number of cells for the sustained spontaneous cAMP oscillations. Corresponding movie files can be found in the supplements of Reference [9].

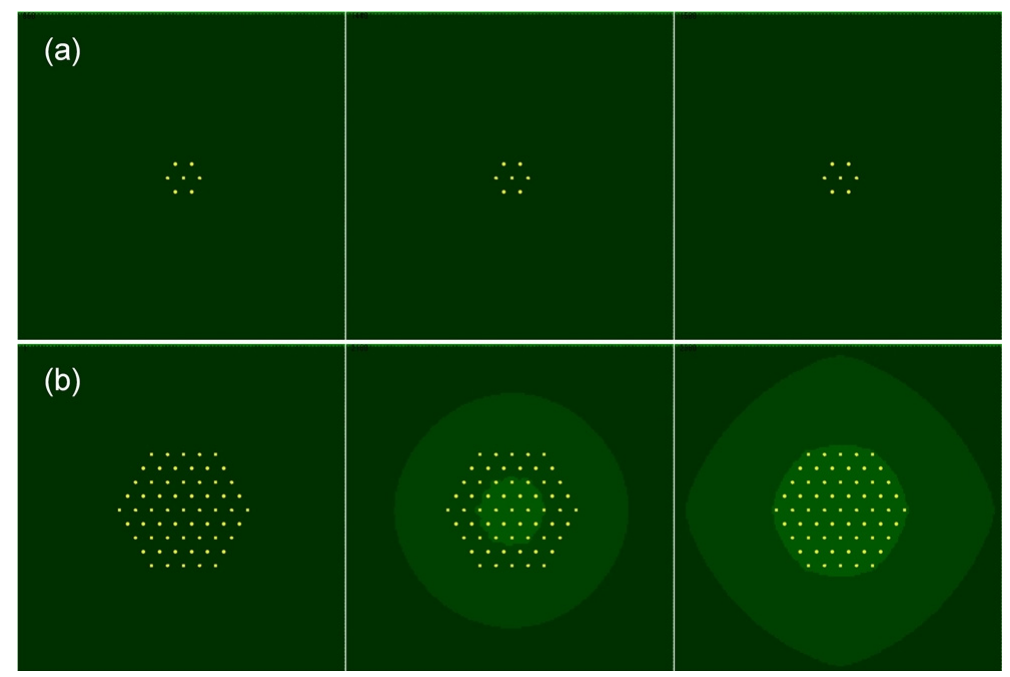

Fig. A-1. Spontaneous cAMP oscillations appear when number of cells $N \geq$ 61. (a) $N=7$, and (b) $N=61$.

\section{References}

[1] D.A. Lauffenburger and J.J. Linderman, Receptors, Oxford University Press, Oxford, UK, 1993.

[2] S.J. Allen, S.E. Crown, and T.M. Handel, Annu. Rev. Immunol, vol. 25, pp. 787-820, 2007.

[3] A.N.R. Weber, M.C. Moncrieffe, M. Gangloff, J. Imler, and N.J. Gay, J. Biol. Chem., vol. 280, pp. 22793-22799, 2005.

[4] V. Sourjik and J.P. Armitage, EMBO J., vol. 29, pp. 2724-2733, 2010.

[5] J.S. Parkinson, P. Ames, and C.A. Studdert, Curr Opin Microbiol, vol. 8, pp. 116-121, 2005.

[6] V. Sourjik and H.C. Berg, PNAS, vol. 99, pp. 123-127, 2002.

[7] R.H. Kessin, Dictyostelium: Evolution, Cell Biology, and the Development of Multicellularity, Cambridge University Press, Cambridge, UK, 2010.

[8] S. Sakurai and S.J. Nagano, Theor. Biol., vol. 307, pp. 37-41, 2012.

[9] S. Nagano and S. Sakurai, Phys. Rev., vol. E88, p. 062710, 2013.

[10] S. Nagano, Phys. Rev. Lett., vol. 80, pp. 4826-4829, 1998.

[11] S. Nagano, Phys. Rev., vol. E67, pp. 0562151-0562154, 2003.

[12] A. Yokoyama and S.J. Nagano, Phys. Soc. Jpn 2008, vol. 77, pp. 0240021-0240029, 2008. 